\title{
UNIVERSAL BOUNDS ON SPECTRAL MEASURES OF ONE-DIMENSIONAL SCHRÖDINGER OPERATORS
}

\author{
CHRISTIAN REMLING
}

\begin{abstract}
Let $H=-d^{2} / d x^{2}+V(x)$ be a Schrödinger operator on $L_{2}(0, \infty)$ with spectral measure $\rho$, and suppose that the potential $V$ is known on an initial interval $[0, N]$. We show that this information yields strong restrictions on $\rho(I)$ for intervals $I \subset \mathbb{R}$. More precisely, we prove upper and lower bounds on $\rho(I)$. The upper bound is finite for any $I$ that is bounded above and the lower bound is positive if the interior of $I$ contains at least two eigenvalues of the operator on $L_{2}(0, N)$. These results are developments of classical work of Chebyshev and Markov on orthogonal polynomials.
\end{abstract}

\section{INTRODUCTION}

We consider one-dimensional Schrödinger equations,

$$
-y^{\prime \prime}(x)+V(x) y(x)=E y(x), \quad 0 \leq x<\infty,
$$

and the associated self-adjoint operators $H_{\alpha}=-d^{2} / d x^{2}+V(x)$ on $L_{2}(0, \infty)$. The index $\alpha \in[0, \pi)$ refers to the boundary condition

$$
y(0) \cos \alpha-y^{\prime}(0) \sin \alpha=0 .
$$

In fact, the dependence on $\alpha$ will usually not be made explicit, as $\alpha$ will remain fixed (but arbitrary) throughout this note. The potential $V$ is assumed to be locally integrable on $[0, \infty)$.

We want to address the following question (which was pointed out to me by David Pearson): Suppose that an initial piece of the potential $V(x), 0 \leq x \leq N$, is known. What, if anything, can then be said about the spectral measure $\rho(I)$ of intervals $I \subset \mathbb{R}$ ? Apart from the intrinsic interest, such considerations are also relevant to the numerical computation of spectral measures.

It is clearly not possible to say anything about the type of $\rho$ (absolutely continuous, singular continuous, or point measure or a mixture of these). For example, the absolutely continuous part of $\rho$ is, up to equivalence, independent of the behavior of the potential on compact sets. It is obviously not even clear if $V$ will be in the limit point or limit circle case at infinity. Nevertheless, we will show that $V$ on $[0, N]$ basically determines $\rho$ on a length scale that is given by the distance between the eigenvalues of the problems on $[0, N]$.

Given $V$ on $[0, N]$, we will introduce a certain family of measures in Sect. 2, which we will call $\mathcal{M}_{N}$. The following property of $\mathcal{M}_{N}$ is especially important: For an arbitrary (locally integrable) extension of $V$ to $[0, \infty)$, the spectral measure(s)

Date: September 20, 2002.

Key words and phrases. Schrödinger operator, spectral measure.

2000 Mathematics Subject Classification. Primary 34L15 34L40; secondary 34L16.

Remling's work was supported by the Heisenberg program of the Deutsche Forschungsgemeinschaft. 
of the corresponding half-line problem will belong to $\mathcal{M}_{N}$. (In fact, $\mathcal{M}_{N}$ is a much larger set.)

For every $\lambda \in \mathbb{R}$, there is a unique boundary condition $\beta \in[0, \pi)$ so that $\lambda$ is an eigenvalue of the problem on $[0, N]$ with boundary condition (1.2) at $x=0$ and

$$
y(N) \cos \beta-y^{\prime}(N) \sin \beta=0
$$

at $x=N$. Let $\mu_{\lambda}$ be the spectral measure of this problem (see again Sect. 2 for a more detailed description).

Theorem 1.1. For all $\lambda \in \mathbb{R}$, we have

$$
\begin{aligned}
\mu_{\lambda}((-\infty, \lambda]) & =\max _{\rho \in \mathcal{M}_{N}} \rho((-\infty, \lambda]), \\
\mu_{\lambda}((-\infty, \lambda)) & =\min _{\rho \in \mathcal{M}_{N}} \rho((-\infty, \lambda)) .
\end{aligned}
$$

This is an analog of classical results of Chebyshev and Markov on orthogonal polynomials - see [1, Sect. 2.5] for these results.

As an illustration, let us fix Neumann boundary conditions at the origin (so $\alpha=\pi / 2$ in (1.2)) and suppose that $V=0$ on $[0,1]$. Then Theorem 1.1 for example yields the following estimate: For any half-line problem, no matter how $V$ behaves on $[1, \infty)$, the spectral measure $\rho$ satisfies $\rho((-\infty, 0]) \leq 1$. Note also that without knowing $V$ on $[0,1]$, one can only say that $\rho((-\infty, 0])<\infty$.

Since $\rho([a, b])=\rho((-\infty, b])-\rho((-\infty, a))$, Theorem 1.1 also implies estimates on the spectral measures of bounded intervals.

Corollary 1.2. Let $\lambda_{i}, \lambda_{j}$ be both eigenvalues of (1.1) on an interval $[0, N]$ with boundary conditions (1.2), (1.3), and let $\rho_{0}$ be the spectral measure of this problem. Then

$$
\begin{aligned}
\rho_{0}\left(\left[\lambda_{i}, \lambda_{j}\right]\right) & =\max _{\rho \in \mathcal{M}_{N}} \rho\left(\left[\lambda_{i}, \lambda_{j}\right]\right), \\
\rho_{0}\left(\left(\lambda_{i}, \lambda_{j}\right)\right) & =\min _{\rho \in \mathcal{M}_{N}} \rho\left(\left(\lambda_{i}, \lambda_{j}\right)\right)
\end{aligned}
$$

In particular, we get a positive lower bound on the spectral measure of an interval whose closure contains three eigenvalues. This last statement can be improved.

Theorem 1.3. Let $I \subset \mathbb{R}$ be an interval, and suppose that for some $\beta \in[0, \pi)$, the interior of $I$ contains an eigenvalue of $(1.1)$ on $[0, N]$ with boundary conditions (1.2), (1.3) and the closure of I contains two eigenvalues of this problem. Then $\inf _{\rho \in \mathcal{M}_{N}} \rho(I)>0$.

In particular, $\inf _{\rho \in \mathcal{M}_{N}} \rho(I)>0$ if $I$ is an open interval containing two eigenvalues of the problem on $[0, N]$ for some boundary condition at $x=N$.

This also follows from Theorem 1.1, but depends on a more careful analysis of the function $\lambda \mapsto \mu_{\lambda}((-\infty, \lambda])$. Theorem 1.3 is sharp: If $I$ is an open interval that does not contain two eigenvalues for some boundary condition (1.3) at $x=N$, then there is a measure $\rho \in \mathcal{M}_{N}$ with $\rho(I)=0$. In fact, given the precise definition of $\mathcal{M}_{N}$ from Sect. 2, this is trivial because in the situation described above, there is a boundary condition at $x=N$ for which $I$ contains no eigenvalue, and the corresponding spectral measure gives zero weight to $I$.

To prove Theorem 1.1, we will basically follow the simple but surprisingly effective strategy from [1, Sect. 2.5]. As an important additional ingredient, we need the Paley-Wiener type description of the spaces of the spectral representation which follows from the theory of de Branges spaces. This topic will be discussed in the next 
section. The proof of Theorem 1.1 is then given in Sect. 3. Theorem 1.3 is proved in Sect. 4. Finally, in Sect. 5 , we derive an asymptotic estimate on $\rho\left(\left(-\infty,-\kappa^{2}\right]\right)$ from Theorem 1.1.

\section{Spectral Representation of Schrödinger operators}

Fix $N>0$ and $\alpha \in[0, \pi)$ and consider the Schrödinger operator $H_{\beta}$ on $L_{2}(0, N)$ with boundary conditions $(1.2),(1.3) . H_{\beta}$ is self-adjoint and has purely discrete spectrum, so the eigenvectors of $H_{\beta}$ form an orthonormal basis of $L_{2}(0, N)$. Thus a spectral representation of $H_{\beta}$ can be obtained as follows. Let $u(x, E)$ be the solution of the Schrödinger equation (1.1) with the initial values $u(0, E)=\sin \alpha$, $u^{\prime}(0, E)=\cos \alpha$. In particular, $u(\cdot, E)$ satisfies the boundary condition (1.2) at $x=0$. Define

$$
\rho_{\beta}=\sum\left\|u\left(\cdot, \lambda_{n}^{(\beta)}\right)\right\|_{L_{2}(0, N)}^{-2} \delta_{\lambda_{n}^{(\beta)}} .
$$

Here, $\delta_{\lambda}$ denotes the Dirac measure at $\lambda$ (so $\left.\delta_{\lambda}(\{\lambda\})=1, \delta_{\lambda}(\mathbb{R} \backslash\{\lambda\})=0\right)$, and the sum is over the eigenvalues $\lambda_{n}^{(\beta)}$ of $H_{\beta}$. Note that $\lambda$ is such an eigenvalue precisely if $u(N, \lambda) \cos \beta-u^{\prime}(N, \lambda) \sin \beta=0 . \rho_{\beta}$ is called the spectral measure of this problem.

Now the map $U$ defined by

$$
(U f)(\lambda)=\int u(x, \lambda) f(x) d x
$$

maps $L_{2}(0, N)$ unitarily onto $L_{2}\left(\mathbb{R}, d \rho_{\beta}\right)$. This is just another way of saying that the eigenvectors form an orthonormal basis because $U$ computes the scalar product of $u(\cdot, \lambda)$ and $f$. The weights in the definition of $\rho_{\beta}$ compensate the fact that the functions $u\left(\cdot, \lambda_{n}^{(\beta)}\right)$ are not normalized.

We use this property of $U$ to define the family $\mathcal{M}_{N}$. A positive Borel measure $\rho$ on $\mathbb{R}$ belongs to $\mathcal{M}_{N}$ if $U$ maps $L_{2}(0, N)$ isometrically into $L_{2}(\mathbb{R}, d \rho)$. $\mathcal{M}_{N}$ depends on $V$ on $(0, N)$ and the boundary condition $(1.2)$ at $x=0$ only. Note that in the definition of $\mathcal{M}_{N}$, we do not require $U$ to be onto (and thus unitary); in fact, $U L_{2}(0, N)=L_{2}(\mathbb{R}, d \rho)$ precisely if $\rho=\rho_{\beta}$ for some $\beta \in[0, \pi)$. Similarly, a spectral measure of a half-line problem is, by definition, a measure $\rho$ for which $U$ is an isometry from $L_{2}(0, N)$ to $L_{2}(\mathbb{R}, d \rho)$ for all $N>0$. Then $U$ of course extends to an isometry from $L_{2}(0, \infty)$ to $L_{2}(\mathbb{R}, d \rho)$. If $V$ is in the limit point case at infinity, there is precisely one spectral measure, and this unique measure can be obtained from the classical Weyl construction (see [4, Chapter 9]). In this case, $U$ automatically maps $L_{2}(0, \infty)$ onto $L_{2}(\mathbb{R}, d \rho)$. In the limit circle case, the measures from the Weyl circle construction are again spectral measures in this wider sense, but there are many other spectral measures. The set of all spectral measures admits a Nevanlinna type description.

It is important to keep in mind that if $\rho$ is a spectral measure of a half-line problem (as defined above or in the narrower sense of Weyl theory), then $\rho \in \mathcal{M}_{N}$ for all $N>0$. In particular, Theorem 1.1 really addresses the question raised at the beginning of this paper.

The measures from $\mathcal{M}_{N}$ are sometimes also called spectral measures of the problem on $[0, N]$ (for example in $[5,7]$ ), but here it is better to avoid this usage of the term, in order to avoid confusion with the spectral measures $\rho_{\beta}, \beta \in[0, \pi)$, introduced above, which form a (small) subset of $\mathcal{M}_{N}$.

This (very classical) material on the spectral representation of $H_{\beta}$ also admits a more function theoretic approach. Here, the crucial tool is the notion of de Branges 
spaces. We will not attempt to give an introduction to this subject here; rather, we will just quote the fact we will need in the next section. For background information and the proof of Theorem 2.1 below, please refer to [7]. The main source for the general theory of de Branges spaces is [3].

Define

$$
S_{N}=\left\{F(z)=\int u(x, z) f(x) d x: f \in L_{2}(0, N)\right\}
$$

so, $S_{N}$ is the image of $L_{2}(0, N)$ under $U$. However, there is a new twist here in that we view $S_{N}$ as a space of entire functions. Let $E_{N}(z)=u(N, z)+i u^{\prime}(N, z)$. The socalled de Branges function $E_{N}$ can now be used to give the following Paley-Wiener type characterization of the de Branges space $S_{N}$.

Theorem 2.1. $F: \mathbb{C} \rightarrow \mathbb{C}$ is in $S_{N} \Longleftrightarrow F$ is entire, $\int_{\mathbb{R}}\left|\frac{F(\lambda)}{E_{N}(\lambda)}\right|^{2} d \lambda<\infty$, and there is a constant $C=C_{F}$ so that $\left|F(z) / E_{N}(z)\right|,\left|F^{\#}(z) / E_{N}(z)\right| \leq C(\operatorname{Im} z)^{-1 / 2}$ for all $z \in \mathbb{C}$ with $\operatorname{Im} z>0$.

Here, as usual, $F^{\#}(z)=\overline{F(\bar{z})}$. Theorem 2.1 follows from the material of [7, Sect. 2, 3], especially [7, Eq. (3.8)]. The statement of Theorem 2.1 may look rather unusual at first sight, but it really is a very natural by-product of the theory of de Branges spaces. It is also similar to the classical Theorem of Paley and Wiener which states that the Fourier transforms of functions from $L_{2}(-a, a)$ are precisely those entire functions which are square integrable on the real line and of exponential type at most $a$. In fact, Theorem 2.1 basically contains the Paley-Wiener Theorem as a special case $(V \equiv 0)$.

Finally, let us note that if $F \in S_{N}$, then $\int_{\mathbb{R}}|F(\lambda)|^{2} d \rho(\lambda)$ has the same finite value for all $\rho \in \mathcal{M}_{N}$. Indeed, this follows immediately from the defining property of the measures from $\mathcal{M}_{N}$. So Theorem 2.1 gives a convenient description of the functions that are integrated correctly by all measures from $\mathcal{M}_{N}$. In fact, the set $S_{N}$ is independent of the potential $V[7$, Theorem 4.1] and it is thus possible to give an even more explicit description of $S_{N}$ (which does not involve, via $E_{N}$, the unknown functions $\left.u(N, z), u^{\prime}(N, z)\right)$. However, for our purposes, Theorem 2.1 is more useful.

\section{Proof of Theorem 1.1}

Fix $\lambda \in \mathbb{R}$, and determine the (unique) boundary condition $\beta$ for which $\lambda$ is an eigenvalue of $H_{\beta}$. Here, $H_{\beta}$ still denotes the operator on $L_{2}(0, N)$ with boundary conditions (1.2), (1.3). Let $\lambda_{1}<\lambda_{2}<\cdots$ be the complete list of eigenvalues of $H_{\beta}$, where, let us say, $\lambda=\lambda_{k}$.

Let $p_{n}(n>k)$ be the unique polynomial of degree $2 n-2$ with

$$
\begin{array}{ll}
p_{n}\left(\lambda_{i}\right)=1 & (i=1, \ldots, k), \\
p_{n}\left(\lambda_{i}\right)=0 & (i=k+1, \ldots, n), \\
p_{n}^{\prime}\left(\lambda_{i}\right)=0 & (i=1, \ldots, n, i \neq k) .
\end{array}
$$

Existence of $p_{n}$ can be proved by giving an explicit Lagrange type formula. To do this, put

$$
g_{j}(\lambda)=\left(\lambda-\lambda_{k}\right) \prod_{i \neq k, j}\left(\lambda-\lambda_{i}\right)^{2} \quad(j=1, \ldots, k-1)
$$


$f_{j}(\lambda)=\left(a_{j} \lambda+b_{j}\right) g_{j}(\lambda)$. It is easy to see that $a_{j}, b_{j}$ can be chosen so that $f_{j}\left(\lambda_{j}\right)=1$, $f_{j}^{\prime}\left(\lambda_{j}\right)=0$; here, one uses the fact that $g_{j}\left(\lambda_{j}\right) \neq 0$. So $f_{j}$ is a polynomial of degree $2 n-2$ with $f_{j}\left(\lambda_{i}\right)=\delta_{i j}, f_{j}^{\prime}\left(\lambda_{i}\right)=0(i \neq k)$. For $j=k$, the construction of a polynomial with these properties is even easier: simply let

$$
f_{k}(\lambda)=\prod_{i \neq k} \frac{\left(\lambda-\lambda_{i}\right)^{2}}{\left(\lambda_{k}-\lambda_{i}\right)^{2}}
$$

Now $p_{n}=\sum_{j=1}^{k} f_{j}$ is the sought polynomial. Uniqueness of $p_{n}$ is clear (and not needed here) because the difference of two such polynomials has $2 n-1$ zeros, counting multiplicities.

Next, we claim that $p_{n} \geq \chi_{\left(-\infty, \lambda_{k}\right]}$. To show this, note that $p_{n}^{\prime}$ has a zero in each of the intervals $\left(\lambda_{1}, \lambda_{2}\right), \ldots,\left(\lambda_{k-1}, \lambda_{k}\right),\left(\lambda_{k+1}, \lambda_{k+2}\right), \ldots,\left(\lambda_{n-1}, \lambda_{n}\right)$. This follows from Rolle's Theorem. Moreover, $p_{n}^{\prime}\left(\lambda_{i}\right)=0(i=1, \ldots, k-1, k+1, \ldots, n)$. We have just recorded $2 n-3$ zeros of $p_{n}^{\prime}$, but the degree of $p_{n}^{\prime}$ is $2 n-3$, so we have the complete list of zeros of $p_{n}^{\prime}$, and all these zeros are simple. It follows that $p_{n}$ is decreasing in $\left[\lambda_{k}, \lambda_{k+1}\right]$ because $p_{n}\left(\lambda_{k}\right)=1, p_{n}\left(\lambda_{k+1}\right)=0$, and $p_{n}^{\prime}$ has no zero in $\left(\lambda_{k}, \lambda_{k+1}\right)$. It also follows that $p_{n}$ has local minima precisely at the $\lambda_{i}$ with $i \neq k$ (and $i \leq n$ ) and no maxima outside $\left[\lambda_{1}, \lambda_{n}\right]$. Thus the asserted inequality holds.

Introduce $q_{n}$ by writing $p_{n}$ as

$$
p_{n}(\lambda)=q_{n}(\lambda) \prod_{j=k+1}^{n}\left(1-\frac{\lambda}{\lambda_{j}}\right)^{2} .
$$

Obviously, this must be modified if one of the $\lambda_{j}$ 's equals zero. In this case, we replace $\left(1-\lambda / \lambda_{j}\right)^{2}$ by $\lambda^{2}$. In the sequel, we will simply assume that $\lambda_{j} \neq 0$ for all $j$. The general case can immediately be reduced to this situation by adding a suitable constant to the potential $V$.

So $q_{n}$ is a polynomial of degree $2 k-2$. The function

$$
U(\lambda):=u(N, \lambda) \cos \beta-u^{\prime}(N, \lambda) \sin \beta
$$

has zeros precisely at the eigenvalues $\left\{\lambda_{j}\right\} . U$ is an entire function of order $1 / 2$ (see [6, Chapter 1, Theorem 3]). This means that $|U(z)| \lesssim e^{|z|^{1 / 2+\epsilon}}$ for all positive $\epsilon$, but for no negative $\epsilon$. So the Hadamard factorization of $U$ reads

$$
U(z)=c \prod_{j=1}^{\infty}\left(1-\frac{\lambda}{\lambda_{j}}\right) .
$$

Since $U$ is real on the real line, $c \in \mathbb{R}$. See [2] for background information on the Hadamard factorization.

We now see that if $n \rightarrow \infty$, the products from (3.1) converge locally uniformly to the function

$$
\frac{U^{2}(\lambda)}{c^{2} \prod_{j=1}^{k}\left(1-\frac{\lambda}{\lambda_{j}}\right)^{2}} .
$$

Moreover, the polynomials $q_{n}$ also converge locally uniformly to a limiting polynomial $q$ of degree $2 k-2$. To see this, we proceed as in the existence proof for the $p_{n}$ 's. Denote the product from (3.1) by $r_{n}(\lambda)$. Then the polynomials $q_{n}$ satisfy

$$
\begin{gathered}
r_{n}\left(\lambda_{i}\right) q_{n}\left(\lambda_{i}\right)=1 \quad(i=1, \ldots, k), \\
r_{n}^{\prime}\left(\lambda_{i}\right) q_{n}\left(\lambda_{i}\right)+r_{n}\left(\lambda_{i}\right) q_{n}^{\prime}\left(\lambda_{i}\right)=0 \quad(i=1, \ldots, k-1),
\end{gathered}
$$


and, conversely, these conditions uniquely determine the $q_{n}$ 's: Construct polynomials $f_{j}, g_{j}$ of degree $2 k-2$ with $f_{j}\left(\lambda_{i}\right)=\delta_{i j}(i \leq k), f_{j}^{\prime}\left(\lambda_{i}\right)=0(i<k)$ and $g_{j}\left(\lambda_{i}\right)=0(i \leq k), g_{j}^{\prime}\left(\lambda_{i}\right)=\delta_{i j}(i<k)$. Using these functions, we can write $q_{n}$ as

$$
q_{n}(\lambda)=\sum_{i=1}^{k} \frac{1}{r_{n}\left(\lambda_{i}\right)} f_{i}(\lambda)-\sum_{i=1}^{k-1} \frac{r_{n}^{\prime}\left(\lambda_{i}\right)}{r_{n}^{2}\left(\lambda_{i}\right)} g_{i}(\lambda) .
$$

Now $r_{n}\left(\lambda_{i}\right)$ converges to the function from (3.2), evaluated at $\lambda_{i}$. The derivative $r_{n}^{\prime}$ is also convergent (this of course follows automatically from the convergence of $r_{n}$ with the help of Cauchy's integral formula for the derivative), so we conclude that $q_{n}$ converges, as desired.

Since both factors converge, $p_{n}$ itself also converges (locally uniformly) to a limiting function. On the real line, we can write this limiting function in the form

$$
\lim _{n \rightarrow \infty} p_{n}(\lambda)=\overline{F_{1}(\lambda)} F_{2}(\lambda) \quad(\lambda \in \mathbb{R}) .
$$

Here,

$$
F_{i}(\lambda)=\frac{U(\lambda)}{c \prod_{j=1}^{k}\left(1-\frac{\lambda}{\lambda_{j}}\right)} s_{i}(\lambda),
$$

where $s_{i}$ is a polynomial of degree $k-1$. In fact, this just summarizes what we have proved above, provided that $\overline{s_{1}(\lambda)} s_{2}(\lambda)=q(\lambda)(\lambda \in \mathbb{R})$. To obtain such a factorization of $q$, we only need to distribute the linear factors of $q$ evenly among the two factors $\overline{s_{1}}, s_{2}$ in an arbitrary way.

By passing to the limit in the inequality $p_{n} \geq \chi_{\left(-\infty, \lambda_{k}\right]}$, we obtain

$$
\overline{F_{1}(\lambda)} F_{2}(\lambda) \geq \chi_{\left(-\infty, \lambda_{k}\right]}(\lambda) \quad(\lambda \in \mathbb{R}) .
$$

Moreover, since $p_{n}\left(\lambda_{i}\right)=\chi_{\left(-\infty, \lambda_{k}\right]}\left(\lambda_{i}\right)$ for $i=1, \ldots, n$, we have equality at the eigenvalues $\lambda_{i}$ :

$$
\overline{F_{1}\left(\lambda_{i}\right)} F_{2}\left(\lambda_{i}\right)=\chi_{\left(-\infty, \lambda_{k}\right]}\left(\lambda_{i}\right) \quad(i \in \mathbb{N}) .
$$

Next, we claim that $F_{i} \in S_{N}$ for $i=1,2$. To prove this, we use Theorem 2.1. Obviously, $F_{i}$ is entire. For large $|\lambda|$, we have the estimate $\left|F_{i}(\lambda)\right| \lesssim \frac{|U(\lambda)|}{|\lambda|}$, and $|U(\lambda)| \leq\left|E_{N}(\lambda)\right|$ for all $\lambda \in \mathbb{R}$, so $\int_{\mathbb{R}}\left|F_{i} / E_{N}\right|^{2}<\infty$. To verify the third condition from Theorem 2.1, we use the fact that $\operatorname{Im} \frac{u^{\prime}(N, z)}{u(N, z)}<0$ if $\operatorname{Im} z>0$. This is well known and can be seen by evaluating $\int_{0}^{N}|u(x, z)|^{2} d x$ with the help of Green's identity. As a consequence, $|u(N, z)|^{2}+\left|u^{\prime}(N, z)\right|^{2}<\left|E_{N}(z)\right|^{2}$ if $\operatorname{Im} z>0$ and hence $|U(z)|<\left|E_{N}(z)\right|$ for these $z$. Thus $\left|F_{i}(z) / E_{N}(z)\right| \lesssim|z|^{-1}$ for large $z \in \mathbb{C}$ with positive imaginary part. Since $U^{\#}=U$, we also have that $\left|F_{i}^{\#}(z) / E_{N}(z)\right| \lesssim|z|^{-1}$. If $|z| \geq 1$, then $|z|^{-1} \leq|z|^{-1 / 2} \leq|\operatorname{Im} z|^{-1 / 2}$, as required. For small $|z|$, there is of course nothing to prove.

Now recall the definitions from the beginning of this section. Denote the spectral measure of $H_{\beta}$ by $\rho_{\beta}$. Let $\rho \in \mathcal{M}_{N}$ be arbitrary. Note that $\left\{\lambda_{i}: i \in \mathbb{N}\right\}$ supports $\rho_{\beta}$, so $(3.3)$ says that $\overline{F_{1}} F_{2}=\chi_{\left(-\infty, \lambda_{k}\right]} \rho_{\beta}$-almost everywhere. Therefore,

$$
\begin{aligned}
\rho\left(\left(-\infty, \lambda_{k}\right]\right) & =\int \chi_{\left(-\infty, \lambda_{k}\right]} d \rho \leq \int \overline{F_{1}} F_{2} d \rho \\
& =\int \overline{F_{1}} F_{2} d \rho_{\beta}=\int \chi_{\left(-\infty, \lambda_{k}\right]} d \rho_{\beta}=\rho_{\beta}\left(\left(-\infty, \lambda_{k}\right]\right) .
\end{aligned}
$$


The second equality holds because $F_{i} \in S_{N}$, so, as observed at the end of Sect. 2, $\int\left|F_{i}\right|^{2} d \rho$ is independent of $\rho \in \mathcal{M}_{N}$. By polarization, $\int \overline{F_{1}} F_{2} d \rho$ then also has the same value for all $\rho \in \mathcal{M}_{N}$. So we may use the measure $\rho_{\beta} \in \mathcal{M}_{N}$ to evaluate this integral. Since $\rho_{\beta}=\mu_{\lambda_{k}}$ if the notation from Theorem 1.1 is used, we have proved the first part of Theorem 1.1. The proof of the second part is analogous.

\section{Proof of Theorem 1.3}

We will prove below (see Theorem 4.1) that the function $\lambda \mapsto \mu_{\lambda}((-\infty, \lambda])$ is strictly increasing. Theorem 1.3 follows rather quickly from this fact.

To fix ideas, let us assume that the interval $I$ from the statement of Theorem 1.3 is of the form $I=\left(\lambda_{k}-\delta, \lambda_{k+1}\right)$, where $\delta>0$ and $\lambda_{k}, \lambda_{k+1}$ are consecutive eigenvalues for some (fixed, but arbitrary) boundary condition (1.3) at $x=N$. Then Theorem 1.1 shows that for all $\rho \in \mathcal{M}_{N}$,

$$
\begin{aligned}
\rho(I) & =\rho\left(\left(-\infty, \lambda_{k+1}\right)\right)-\rho\left(\left(-\infty, \lambda_{k}-\delta\right]\right) \\
& \geq \mu_{\lambda_{k+1}}\left(\left(-\infty, \lambda_{k+1}\right)\right)-\mu_{\lambda_{k}-\delta}\left(\left(-\infty, \lambda_{k}-\delta\right]\right) \\
& =\mu_{\lambda_{k}}\left(\left(-\infty, \lambda_{k}\right]\right)-\mu_{\lambda_{k}-\delta}\left(\left(-\infty, \lambda_{k}-\delta\right]\right),
\end{aligned}
$$

and this is positive by Theorem 4.1 below. The case $I=\left(\lambda_{k}, \lambda_{k+1}+\delta\right)$ is treated similarly, and a general $I$ satisfying the hypotheses of Theorem 1.3 must contain either such an interval or one of the form discussed above.

Theorem 4.1. The function $\lambda \mapsto \mu_{\lambda}((-\infty, \lambda])$ from Theorem 1.1 is strictly increasing, piecewise real analytic and $C^{\infty}$.

These properties of $\mu_{\lambda}((-\infty, \lambda])$ seem to be of some independent interest, so we state more here than needed for the proof of Theorem 1.3.

Proof of Theorem 4.1. Let $\lambda_{1}<\lambda_{2}<\ldots$ be the Dirichlet eigenvalues (that is, $\beta=0$ in (1.3)). For $\lambda \notin\left\{\lambda_{j}\right\}$ and $n \in \mathbb{N}_{0}$, let $E_{n}(\lambda)$ be the solution $E \in\left(\lambda_{n}, \lambda_{n+1}\right)$ $\left(\lambda_{0}:=-\infty\right)$ of

$$
\frac{u^{\prime}(N, E)}{u(N, E)}=\frac{u^{\prime}(N, \lambda)}{u(N, \lambda)}
$$

In other words, $E_{n}(\lambda)$ is chosen so that $u\left(\cdot, E_{n}(\lambda)\right)$ satisfies the same boundary condition as $u(\cdot, \lambda)$. Oscillation theory shows that $E_{n}(\lambda)$ is well defined. In fact, the function $u^{\prime} / u$ is strictly decreasing on $\left(\lambda_{n}, \lambda_{n+1}\right)$. So $E_{n}$ is a real analytic function on each of the intervals $\left(\lambda_{j}, \lambda_{j+1}\right)$ by the implicit function theorem.

Assume that $\lambda \in\left(\lambda_{n}, \lambda_{n+1}\right)$. We then have the following formula for $f(\lambda) \equiv$ $\mu_{\lambda}((-\infty, \lambda]):$

$$
f(\lambda)=\sum_{j=0}^{n} \rho_{\beta}\left(\left\{E_{j}(\lambda)\right\}\right)=\sum_{j=0}^{n}\left(\int_{0}^{N} u^{2}\left(x, E_{j}(\lambda)\right) d x\right)^{-1},
$$

where $\beta \in(0, \pi)$ satisfies $\cot \beta=u^{\prime}(N, \lambda) / u(N, \lambda)$. This representation makes it clear that $f$ is a real analytic function on $\left(\lambda_{n}, \lambda_{n+1}\right)$.

From the role of $f$ as an upper bound for $\rho((-\infty, \lambda])$ in Theorem 1.1, it is immediately clear that $f$ is increasing. To prove that $f$ is strictly increasing, assume the contrary: Then there are $a<b$ with $f(a)=f(b)$. The interval $(a, b)$ will intersect $\left(\lambda_{n}, \lambda_{n+1}\right)$ for some $n \in \mathbb{N}_{0}$. But $f$ is real analytic on $\left(\lambda_{n}, \lambda_{n+1}\right)$, so $f$ is constant on this whole interval. It is easy to see that $f$ is continuous - we will 
prove in a moment that $f \in C^{\infty}$. So $f$ is constant on $\left[\lambda_{n}, \lambda_{n+1}\right]$. This is clearly impossible, because $f\left(\lambda_{n+1}\right)=f\left(\lambda_{n}\right)+\rho_{0}\left(\left\{\lambda_{n+1}\right\}\right)$.

We now come to the most difficult part of the proof: We still have to show that $f \in C^{\infty}(\mathbb{R})$. We know already that $f$ is real analytic on the intervals $\left(\lambda_{n}, \lambda_{n+1}\right)$, so it remains to investigate the behavior of $f$ at the points $\lambda_{n}$. From (4.1) and the discussion preceding this equation, we see that for sufficiently small $h>0$,

$$
f\left(\lambda_{n}+h\right)=\widetilde{f}\left(\lambda_{n}+h\right)+\left(\int_{0}^{N} u^{2}\left(x,-\kappa^{2}(h)\right) d x\right)^{-1} .
$$

Here, $\tilde{f}$ is the analytic continuation of $f$ from $\left(\lambda_{n-1}, \lambda_{n}\right)$, and the energy $-\kappa^{2}(h)<$ $\lambda_{1}$ is defined by the requirement that $u\left(\cdot,-\kappa^{2}(h)\right)$ satisfy the same boundary condition as $u\left(\cdot, \lambda_{n}+h\right)$. In other words, $\kappa(h)$ is the unique solution $\kappa>0,-\kappa^{2}<\lambda_{1}$ of

$$
\frac{u\left(N,-\kappa^{2}\right)}{u^{\prime}\left(N,-\kappa^{2}\right)}=\frac{u\left(N, \lambda_{n}+h\right)}{u^{\prime}\left(N, \lambda_{n}+h\right)} .
$$

Note that (4.2) expresses the fact that $f$ fails to be analytic at $\lambda_{n}$ only because of the emergence of a new term in the sum from (4.1), and this new summand has been recorded separately in (4.2). Call this last term from (4.2) $F(h)$. We will prove that $\lim _{h \rightarrow 0+} F(h)=0$ and the continuously extended $F$ satisfies $F \in C^{\infty}\left(\left[0, h_{0}\right)\right)$ for some $h_{0}>0$. Finally, we will also prove that $F^{(n)}(0)=0$ for all $n \in \mathbb{N}_{0}$. Here, we take one-sided derivatives at $h=0$. These properties of $F$ will of course imply that $f \in C^{\infty}$ at $\lambda_{n}$ also, as desired.

We need the following Lemma, which will be proved below.

\section{Lemma 4.2.}

$$
\begin{gathered}
u\left(x,-\kappa^{2}\right)=\cosh \kappa x+O\left(\frac{e^{\kappa x}}{\kappa}\right), \\
u^{\prime}\left(x,-\kappa^{2}\right)=\kappa \sinh \kappa x+O\left(e^{\kappa x}\right) .
\end{gathered}
$$

These formulae hold uniformly in sets of the form $0 \leq x \leq N, \kappa \geq \kappa_{0}$. Moreover,

$$
\left|\frac{\partial^{n} u}{\partial \kappa^{n}}\left(x,-\kappa^{2}\right)\right| \leq C_{n} e^{\kappa x}, \quad\left|\frac{\partial^{n} u^{\prime}}{\partial \kappa^{n}}\left(x,-\kappa^{2}\right)\right| \leq C_{n} \kappa e^{\kappa x} .
$$

By induction, Lemma 4.2 implies that

$$
\left|\frac{d^{n}}{d \kappa^{n}} \int_{0}^{N} u^{2}\left(x,-\kappa^{2}\right) d x\right| \leq C_{n} \frac{e^{2 \kappa N}}{\kappa}
$$

for all $n \in \mathbb{N}_{0}$ (and with new constants $C_{n}$, of course). Now another induction shows that

$$
\left|\frac{d^{n}}{d \kappa^{n}}\left(\int_{0}^{N} u^{2}\left(x,-\kappa^{2}\right) d x\right)^{-1}\right| \leq C_{n} \kappa e^{-2 \kappa N}
$$

Next, we study the function $\kappa(h)$, which was implicitly defined by (4.3). The right-hand side of (4.3) is in $C^{\infty}\left(-h_{0}, h_{0}\right)$ for some $h_{0}>0$. The left-hand side will be denoted by $g(\kappa)$. Clearly, $g$ is also $C^{\infty}$ for sufficiently large $\kappa$, and by standard oscillation theory, $g$ is strictly decreasing for these $\kappa$. Thus $\kappa=\kappa(h)$ is well defined for small $h>0$ and $\kappa \in C^{\infty}\left(0, h_{0}\right)$, where $h_{0}>0$ must be taken sufficiently small. Moreover, by Lemma 4.2, $g(\kappa)=\kappa^{-1}+O\left(\kappa^{-2}\right)$. Since the right-hand side of (4.3) 
has a positive derivative at $h=0$ (this also follows from oscillation theory or, alternatively, from a computation), we obtain, after some standard manipulations with asymptotic expressions, the formula

$$
\kappa(h)=\frac{c}{h}+O(1) \quad(c>0) .
$$

We have that $d g / d \kappa=\left(u_{\kappa} u^{\prime}-u u_{\kappa}^{\prime}\right) /{u^{\prime}}^{2}$; here, an index $\kappa$ indicates partial differentiation with respect to $\kappa$. By a computation using Green's identity, the numerator is equal to $-2 \kappa \int_{0}^{N} u^{2}$. Thus Lemma 4.2 shows that $|d g / d \kappa| \gtrsim \kappa^{-2}$. Recalling (4.5), we now see from (4.3) (by taking derivatives with respect to $h$ and solving for $\kappa^{\prime}$ ) that $\left|\kappa^{\prime}(h)\right| \lesssim h^{-2}$. More generally, arguments of this type also allow us to control the higher order derivatives of $\kappa$. The result of this straightforward but somewhat tedious procedure is an estimate of the form

$$
\left|\kappa^{(n)}(h)\right| \leq C_{n} h^{-\alpha_{n}} \quad\left(0<h<h_{0}, n \in \mathbb{N}_{0}\right) .
$$

There is no need to keep track of the optimal exponents $\alpha_{n}$ here.

By using the chain rule and combining this estimate with (4.4), we obtain (inductively) that

$$
\left|F^{(n)}(h)\right| \leq C_{n} e^{-\gamma / h} \quad\left(0<h<h_{0}, n \in \mathbb{N}_{0}\right),
$$

with $\gamma>0$. Now a final inductive argument yields the desired assertion: Clearly, (4.6) with $n=0$ shows that $\lim _{h \rightarrow 0+} F(h)=0$. If, for general $n \in \mathbb{N}_{0}, F^{(n)}(0)=0$, then, since (4.6) shows that $\lim _{h \rightarrow 0+} F^{(n)}(h) / h=0, F^{(n+1)}(0)$ also exists and equals zero.

Proof of Lemma 4.2. The first part is a standard asymptotic formula (see, for example, $\left[6\right.$, Chapter 1 , Theorem 3]); we will only prove the estimates on $\partial^{n} u / \partial \kappa^{n}$ and $\partial^{n} u^{\prime} / \partial \kappa^{n}$.

The derivatives $u_{\kappa} \equiv \partial u / \partial \kappa, u_{\kappa \kappa} \equiv \partial^{2} u / \partial \kappa^{2}$ etc. satisfy the equations

$$
\begin{aligned}
-u_{\kappa}^{\prime \prime}+\left(V+\kappa^{2}\right) u_{\kappa} & =-2 \kappa u, \\
-u_{\kappa \kappa}^{\prime \prime}+\left(V+\kappa^{2}\right) u_{\kappa \kappa} & =-4 \kappa u_{\kappa}-2 u,
\end{aligned}
$$

and so on. Moreover,

for all $n \in \mathbb{N}$.

$$
\frac{\partial^{n} u}{\partial \kappa^{n}}\left(0,-\kappa^{2}\right)=\frac{\partial^{n} u^{\prime}}{\partial \kappa^{n}}\left(0,-\kappa^{2}\right)=0
$$

The variation-of-constants formula says that the solution of the inhomogenous problem $-y^{\prime \prime}+\left(V+\kappa^{2}\right) y=g, y(0)=y^{\prime}(0)=0$ is given by

$$
y(x)=\int_{0}^{x} K(x, t ; \kappa) g(t) d t
$$

where

$$
K(x, t ; \kappa)=\frac{u\left(x,-\kappa^{2}\right) f\left(t,-\kappa^{2}\right)-u\left(t,-\kappa^{2}\right) f\left(x,-\kappa^{2}\right)}{W(u, f)} .
$$

Here, $f$ is an arbitrary solution of the homogenous equation $-y^{\prime \prime}+\left(V+\kappa^{2}\right) y=0$ that is linearly independent of $u$, and $W(u, f)=u f^{\prime}-u^{\prime} f$. Note that this latter combination (the Wronskian of $u$ and $f$ ) is independent of $x$. 
We will take $f$ as an exponentially small solution. More precisely, let $f$ be the solution of $-f^{\prime \prime}+\left(V+\kappa^{2}\right) f=0$ with $f(N)=e^{-\kappa N}, f^{\prime}(N)=-\kappa e^{-\kappa N}$. Define $g(x)=f(x) e^{\kappa x}$. Then $g$ is the unique solution of the integral equation

$$
g(x)=1-\frac{1}{2 \kappa} \int_{x}^{N}\left(e^{2 \kappa(x-t)}-1\right) V(t) g(t) d t
$$

It follows directly from this equation that $\|g\|_{\infty} \leq 2$ if $\kappa \geq\|V\|_{L_{1}(0, N)}$, and hence

$$
\left|f\left(x,-\kappa^{2}\right)\right| \leq 2 e^{-\kappa x} \quad\left(\kappa \geq\|V\|_{1}\right) .
$$

By evaluating at $x=N$, we see that

$$
W(u, f)=-\kappa e^{-\kappa N} \cosh \kappa N-e^{-\kappa N} \kappa \sinh \kappa N+O(1)=-\kappa+O(1) .
$$

So, by (4.9) and again the formula for $u$ from the first part of the Lemma,

$$
|K(x, t ; \kappa)| \lesssim \frac{1}{\kappa} e^{\kappa(x-t)} .
$$

Now the desired estimates on $\partial^{n} u / \partial \kappa^{n}$ follow immediately from (4.7) by induction on $n$.

The argument for $\partial^{n} u^{\prime} / \partial \kappa^{n}$ is similar: one takes derivatives in (4.7), and so it is now necessary to estimate $\partial K(x, t ; \kappa) / \partial x$. Basically, this is done as above. To control $f^{\prime}$, one differentiates (4.8) to obtain

$$
g^{\prime}(x)=-\int_{x}^{N} e^{2 \kappa(x-t)} V(t) g(t) d t .
$$

It follows that if $\kappa \geq\|V\|_{1}$, then $\left\|g^{\prime}\right\|_{\infty} \leq 2\|V\|_{1} \leq 2 \kappa$ and hence $\left|f^{\prime}\left(x,-\kappa^{2}\right)\right| \leq$ $4 \kappa e^{-\kappa x}$. This in turn implies that $\left|K_{x}(x, t ; \kappa)\right| \lesssim e^{\kappa(x-t)}$, and the claim now follows as before.

\section{A Universal ASYMPtOtic ESTimate}

Theorem 1.1 shows that the potential on an initial interval has a strong influence on the spectral measure above a certain length scale. In fact, this information basically determines the spectral measure of intervals that contain several eigenvalues of the restricted problem. In this section, we will use Theorem 1.1 to prove a universal estimate on $\rho\left(\left(-\infty,-\kappa^{2}\right]\right)$ for large $\kappa$, in order to further illustrate the result.

It is well known (see, for example, [5, Theorem 2.4.2]) that

$$
\lim _{\kappa \rightarrow \infty} \rho\left(\left(-\infty,-\kappa^{2}\right]\right) e^{N \kappa}=0 \quad \forall N>0
$$

if $\rho$ is a spectral measure of a half-line problem. We will prove the following more explicit statement. We will only treat the case of Neumann boundary conditions at the origin $\left(y^{\prime}(0)=0\right.$ or, in other words, $\alpha=\pi / 2$ in $\left.(1.2)\right)$. However, the same method works for arbitrary boundary conditions. In fact, one can also prove two-sided estimates on $\rho\left(\left(k^{2}, l^{2}\right)\right)(k, l$ large) along these lines.

Theorem 5.1. Let $\rho$ be a spectral measure of a half-line problem with Neumann boundary conditions at $x=0$. If $N \geq 1, \kappa \geq 8 \exp \left(\int_{0}^{N}|V(x)| d x\right)$, then

$$
\rho\left(\left(-\infty,-\kappa^{2}\right]\right) \leq 17 \kappa e^{-2 N \kappa}
$$

Since $N$ can be taken arbitrarily large here (provided $\kappa$ is large enough), (5.1) follows immediately from Theorem 5.1. 
Proof. Our starting point is the estimate

$$
|u(x, \lambda)-\cos \sqrt{\lambda} x| \leq \frac{1}{|\lambda|^{1 / 2}} e^{|\operatorname{Im} \lambda|^{1 / 2} x+\int_{0}^{x}|V(t)| d t} .
$$

This is a quantitative version of the first part of Lemma 4.2 - see also [6, Chapter 1, Theorem 3] (note, however, that in [6] it is assumed that $V \in L_{2}(0, x)$, but the same proof works in the general case $\left(V \in L_{1}(0, x)\right)$ as well).

In particular, we have that for $\kappa>0$ and $0 \leq x \leq N$,

$$
u\left(x,-\kappa^{2}\right) \geq \cosh \kappa x-\frac{1}{\kappa} e^{\kappa x+V_{x}} \geq \frac{1}{2} e^{\kappa x}-\frac{1}{\kappa} e^{\kappa x+V_{N}} .
$$

Here, we have used the abbreviation $V_{x}=\int_{0}^{x}|V(t)| d t$. It follows that

$$
\int_{0}^{N} u^{2}\left(x,-\kappa^{2}\right) d x \geq\left(\frac{1}{4}-\frac{e^{V_{N}}}{\kappa}\right) \int_{0}^{N} e^{2 \kappa x} d x=\frac{e^{2 \kappa N}-1}{8 \kappa}\left(1-\frac{4 e^{V_{N}}}{\kappa}\right) .
$$

So, if $\kappa \geq 8 e^{V_{N}}$, then

$$
\int_{0}^{N} u^{2}\left(x,-\kappa^{2}\right) d x \geq \frac{e^{2 \kappa N}-1}{16 \kappa} \geq \frac{e^{2 \kappa N}}{17 \kappa} .
$$

The last inequality follows from the fact that $N \geq 1, \kappa \geq 8$. The problem on $[0, N]$ with (let us say) Dirichlet boundary conditions at $x=N(y(N)=0)$ has no eigenvalues in $\left(-\infty,-64 e^{2 V_{N}}\right.$ ] (in fact, $-V_{N}^{2}$ is a lower bound for the smallest eigenvalue). Therfore $-\kappa^{2}$ is the smallest eigenvalue for the boundary condition for which this energy is in the spectrum. Otherwise, we would get a contradiction to oscillation theory. Hence

$$
\mu_{-\kappa^{2}}\left(\left(-\infty,-\kappa^{2}\right]\right)=\mu_{-\kappa^{2}}\left(\left\{-\kappa^{2}\right\}\right)=\left(\int_{0}^{N} u^{2}\left(x,-\kappa^{2}\right) d x\right)^{-1} \leq 17 \kappa e^{-2 \kappa N} .
$$

Theorem 1.1 shows that this estimate holds for any $\rho \in \mathcal{M}_{N}$.

\section{REFERENCES}

[1] N.I. Akhiezer, The Classical Moment Problem, Oliver and Boyd, Edinburgh, 1965.

[2] R.P. Boas, Entire Functions, Academic Press, New York, 1954.

[3] L. de Branges, Hilbert Spaces of Entire Functions, Prentice-Hall, Englewood Cliffs, 1968.

[4] E.A. Coddington and N. Levinson, Theory of Ordinary Differential Equations, McGraw-Hill, New York, 1972

[5] V.A. Marchenko, Sturm-Liouville Operators and Applications, Birkhäuser, Basel, 1986.

[6] J. Pöschel and E. Trubowitz, Inverse Spectral Theory, Academic Press, Orlando, 1987.

[7] C. Remling, Schrödinger operators and de Branges spaces, to appear in J. Funct. Anal. (electronically available on my homepage).

Universität OsnabrüCK, Fachbereich Mathematik/Informatik, 49069 Osnabrück, GerMANY

E-mail address: cremling@mathematik. uni-osnabrueck.de

URL: www.mathematik. uni-osnabrueck.de/staff/phpages/remlingc.rdf.html 